\title{
Photodynamic Therapy Combined with 1064-nm Nd:YAG Laser Therapy and Topical Efinaconazole for Refractory Onychomycosis: Case Series
}

Jae Wan Park

Young Gue Koh

Seong Jun Seo

Kui Young Park

Department of Dermatology, Chung-Ang University Hospital, Chung-Ang University College of Medicine, Seoul, Korea
Received August 5, 2021

Accepted September 7, 2021

\section{Correspondence}

Kui Young Park

Department of Dermatology, Chung-Ang

University Hospital, Chung-Ang University College of Medicine, 102 Heukseok-ro, Dongjak-gu, Seoul 06973, Korea

Tel.: +82-2-6299-1525

Fax: +82-2-6299-1718

E-mail: kykylacaumc.or.kr

(C) Korean Society for Laser Medicine and Surgery

(c) This is an open access article distributed under the terms of the Creative Commons Attribution NonCommercial License (http://creativecommons.org/ licenses/by-nc/4.0) which permits unrestricted noncommercial use, distribution, and reproduction in any medium, provided the original work is properly cited.
Onychomycosis is the most common disease of the nail, with a worldwide prevalence of $5.5 \%$. It causes local pain, paresthesia, and cosmetic problems and decreases the patients' quality of life. Conventional treatments include the administration of topical and systemic agents. However, factors such as subungual hyperkeratosis and biofilm formation may affect the efficacy of these treatments. Moreover, side effects associated with systemic agents are a major concern for patients. Therefore, various novel treatments are being developed; among them, photodynamic therapy (PDT) and Nd:YAG laser are promising, and several studies have demonstrated the efficacy and safety of PDT and laser therapy. Here, we report the efficacy and tolerability of PDT combined with 1064-nm Nd:YAG laser therapy and topical efinaconazole for the treatment of three patients with refractory onychomycosis.

\section{Key words}

Nd:YAG laser; Onychomycosis; Photodynamic therapy 


\section{INTRODUCTION}

Onychomycosis is a fungal nail infection caused by dermatophytes, non-dermatophytes, and yeasts and is the most common nail disorder seen in clinical practice. Due to fungal overgrowth in the nail bed, subungual hyperkeratosis and various nail plate discoloration are typical clinical presentations. ${ }^{1}$

Onychomycosis therapy aims at both eliminating the infecting fungal organism and restoring the nail to its normal state. ${ }^{2}$ However, conventional treatments using topical or systemic agents often encounter some hurdles such as limited penetration of the nail, resistant biofilms, and low treatment compliance. Furthermore, there are concerns associated with oral drugs, such as systemic side effects. ${ }^{3,4}$

Photodynamic therapy (PDT) is a non-invasive treatment that combines light-based modalities such as a light-emitting diode (LED) with photosensitizers. When the applied photosensitizer is excited by visible light, reactive oxygen species and free radicals are generated, which destroy fungal cells. ${ }^{5}$

Several studies have shown that 1064-nm long-pulsed $\mathrm{Nd}$ :YAG lasers may offer a valuable treatment alternative for patients with mild onychomycosis. Although the exact mechanism is unclear, lasers may exert a therapeutic effect by selective photothermolysis. ${ }^{6}$

Herein, we present the cases of three patients with refractory onychomycosis treated with PDT combined with 1064-nm long-pulsed Nd:YAG laser therapy and topical efinaconazole.

\section{CASE REPORT}

\section{Case 1}

A 48-year-old male patient presented with a 1-year history of yellowish discoloration of his big toenail on both feet and a pincer nail on his left big toe (Fig. 1A). He had been diagnosed had applied topical ciclopirox for 1 year, but there was no clinical improvement. We treated him with PDT combined with Nd:YAG laser therapy lexcel V ${ }^{T M}$; Cutera $^{\circledR}$, Brisbane, CA, USAl and topical efinaconazole. First, we performed 1064-nm long-pulsed Nd:YAG laser therapy using the following parameters: $5 \mathrm{~J} / \mathrm{cm}^{2}, 0.3 \mathrm{~ms}$ pulse duration, and $4 \mathrm{~Hz}$ repetition rate. Second, PDT was performed with $16 \%$ methyl aminolevulinate (MAL), which was applied and sealed for 1 hour. Third, MAL-treated areas were irradiated with an LED at a wavelength of 630 $\mathrm{nm}$ at $60 \mathrm{~J} / \mathrm{cm}^{2}$. Each session was separated by a 4-week interval. After four sessions, clinical improvement was observed and the pincer nail on his left big toenail normalized (Fig. 1B).

\section{Case 2}

A 53-year-old female patient presented with a 3-year history of yellowish discoloration and onycholysis of the big toenail on both her feet (Fig. 2A). She had applied topical amorolfine for 1 year and oral terbinafine for 3 months, but the lesions appeared refractory. Because she refused further systemic treatment for onychomycosis,
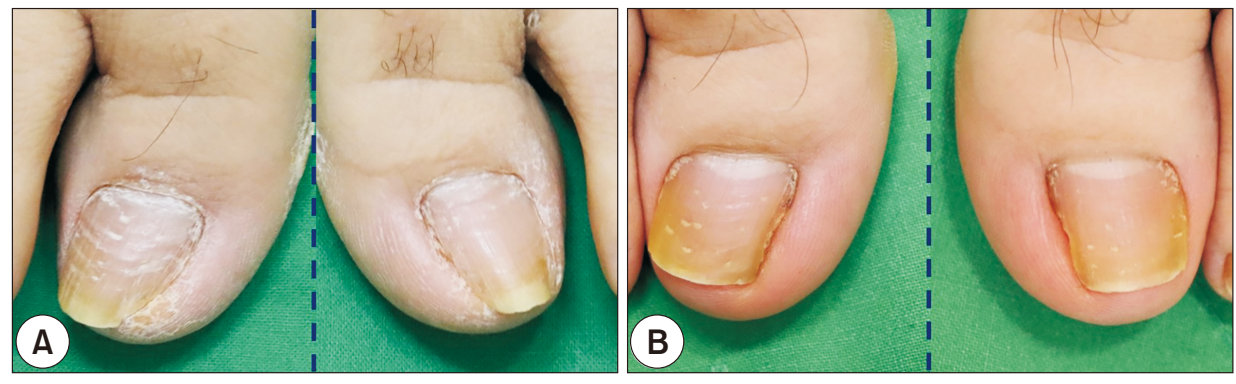

Fig. 1. Case 1: 48-year-old man. (A) Yellowish discoloration and onycholysis of distal nail plate are observed before treatment. Pincer nail on his left big toe is also observed. (B) Clinical improvement after 4 sessions. Remarkable improvement of pincer nail on his left big toe is noted.
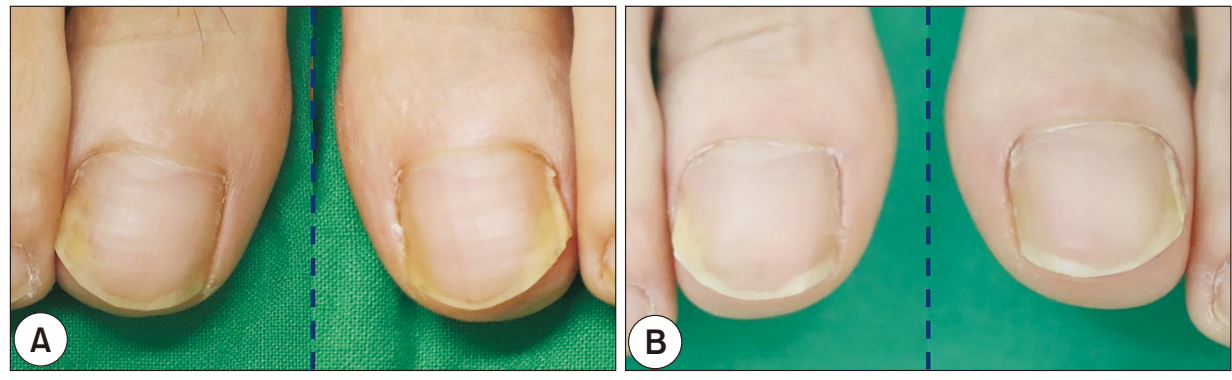

Fig. 2. Case 2: 53-year-old woman (A) Yellowish discoloration and onycholysis are observed before treatment. (B) Clinical improvement is observed after 5 sessions. 


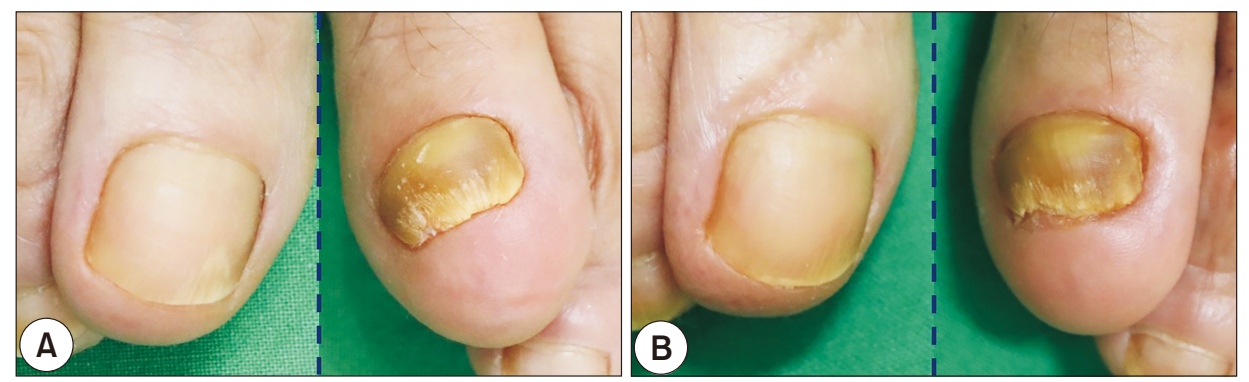

Fig. 3. Case 3: 66-year-old woman. (A) Distal onycholysis on her right big toenail, onycholysis, yellowish discoloration, and subungual hyperkeratosis are observed on her left big toenail before treatment. (B) Clinical improvement is observed on her right big toenail. However, the refractory lesion is observed on her left big toenail after 5 sessions.

we decided to treat her with PDT combined with Nd:YAG laser therapy and topical efinaconazole using the same protocol followed for Case 1. Clinical improvement of the lesions was observed after five sessions (Fig. 2B).

\section{Case 3}

A 66-year-old female patient presented with a 3-year history of yellowish discoloration of the big toenail on both her feet. Severe onycholysis and subungual hyperkeratosis were observed on her left big toenail (Fig. 3A). She had been treated with oral terbinafine and topical efinaconazole for 1 year, but the lesions were refractory. We decided to treat her with PDT combined with Nd:YAG laser therapy and topical efinaconazole, as she refused further oral medication. After five sessions, clinical improvement was observed on her right big toenail. However, there was no significant improvement on her left big toenail (Fig. 3B).

\section{DISCUSSION}

Since Kamp et al. demonstrated the fungicidal effects of PDT on Trichophyton rubrum, several studies have reported the cytotoxic and antimicrobial properties of PDT. ${ }^{7}$ A 1064-nm long- pulsed Nd:YAG laser emits optical energy that can penetrate the skin surface to a depth of $3-5 \mathrm{~mm}$ and coagulate tissue, raising the temperature to $60^{\circ} \mathrm{C}$, which entails protein denaturation and collagen coagulation. ${ }^{8}$ Because 1064-nm Nd:YAG lasers use a longer wavelength than that used in PDT, which enables deeper penetration, combined therapy may be synergistic.

Efficacy of PDT may vary depending on the severity as one clinical study showed a significantly lower clinical response to PDT in patients with severe distal and lateral subungual onychomycosis (DLSO) (63.6\%) than in those with mild to moderate DLSO (100\%). ${ }^{9}$ In our cases, refractory lesions of all three patients showed significant improvement, except for severe onychomycosis of the left big toenail in the 66-year-old female patient (Case 3). We presume that some severe and refractory onychomycosis would require further treatment. On the other hand, it is remarkable that the pincer nail of the 48-year-old male patient (Case 1) showed significant improvement (Fig. 1) because conservative treatment of pincer nail shows high rate of recurrence and often requires long-term treatment and the effect may be modest in cases with severe deformity.

Systemic antifungal agent treatment has demonstrated several concerning side effects along with the necessity of long-term use of agents; therefore, patient compliance to this treatment is an issue. The relatively long interval 14 weeks) of this combined PDT and Nd:YAG laser therapy may be beneficial for those who cannot undergo or show low compliance to systemic antifungal treatment. Furthermore, for maximizing the efficacy of combined treatment, we recommend that patients use topical efinaconazole, which showed the highest cure rates among topical agents.

Some common side effects of PDT and Nd:YAG laser therapy reported were mild pain, burning sensation, erythema, and blistering. There were also photosensitizerrelated problems reported due to the use of methylene blue and toluidine blue; several patients discontinued treatment because of the blue discoloration. ${ }^{10}$ We used MAL as a photosensitizer and reported no side effects.

Our cases demonstrate that PDT combined with 1064$\mathrm{nm} \mathrm{Nd}$ :YAG laser therapy and topical efinaconazole may be an effective alternative treatment for refractory onychomycosis. As the treatment mechanisms of PDT and $\mathrm{Nd}$ :YAG laser are not fully understood, it is not certain that PDT and laser therapies have synergistic effects when used together. Therefore, further well-designed controlled studies should be conducted to establish the optimal regimen, efficacy, and safety of PDT with Nd:YAG 
laser therapy and topical efinaconazole for the treatment of onychomycosis.

\section{CONFLICT OF INTEREST}

No potential conflict of interest relevant to this article was reported.

\section{FUNDING}

None.

\section{REFERENCES}

1. de Berker D. Clinical practice. Fungal nail disease. N Engl J Med 2009;360:2108-16.

2. Gupta AK, Studholme C. How do we measure efficacy of therapy in onychomycosis: patient, physician, and regulatory perspectives. J Dermatolog Treat 2016;27:498-504.

3. Elewski B, Pariser D, Rich P, Scher RK. Current and emerging options in the treatment of onychomycosis. Semin Cutan Med Surg 2013;32(2 Suppl 1):S9-12.

4. Graybill JR, Sharkey PK. Fungal infections and their management. Br J Clin Pract Suppl 1990;71:23-31.

5. Bhatta AK, Keyal U, Wang XL. Photodynamic therapy for ony- chomycosis: a systematic review. Photodiagnosis Photodyn Ther 2016;15:228-35.

6. Liddell LT, Rosen T. Laser therapy for onychomycosis: fact or fiction? J Fungi (Basell) 2015;1:44-54

7. Kamp H, Tietz HJ, Lutz M, Piazena H, Sowyrda P, Lademann J, et al. Antifungal effect of 5-aminolevulinic acid PDT in Trichophyton rubrum. Mycoses 2005;48:101-7.

8. Noguchi H, Miyata K, Sugita T, Hiruma M, Hiruma M. [Treatment of onychomycosis using a 1064nm Nd:YAG laser]. Med Mycol J 2013;54:333-9. Japanese.

9. Souza LW, Souza SV, Botelho AC. Distal and lateral toenail onychomycosis caused by Trichophyton rubrum: treatment with photodynamic therapy based on methylene blue dye. An Bras Dermatol 2014:89:184-6.

10. Tardivo JP, Wainwright M, Baptista M. Small scale trial of photodynamic treatment of onychomycosis in São Paulo. J Photochem Photobiol B 2015;150:66-8.

How to cite this article: Park JW, Koh YG, Seo SJ, Park KY. Photodynamic therapy combined with 1064-nm Nd:YAG laser therapy and topical efinaconazole for refractory onychomycosis: case series. Med Lasers 2021;10:185-188. https://doi. org/10.25289/ML.2021.10.3.185 